\title{
A Survey of Intelligent Decision Support System
}

\author{
He Changlin ${ }^{1, a^{*}}$, Li Yufen ${ }^{2, b}$ \\ ${ }^{1}$ Information Technology Center of Hexi University, Zhangye Gansu 734000 China \\ ${ }^{2}$ ICC of Zhangye Power Supply Company, Gansu 734000 China \\ ahecl@hxu.edu.cn, bliyufen626@163.com
}

\begin{abstract}
IDSS can be generated through combining AI technology and DSS and applying Expert System. The generation and current status of IDSS are firstly expounded in this paper. Then it describes the three types and application scenarios of IDSS in detail, describes the characteristics of IDSS, gives the system structure of IDSS, analyzes and expounds the constitution system of the system structure, analyzes the development trend of IDSS and looks far ahead into the future direction of research on IDSS.
\end{abstract}

Keywords: IDSS, AI, Data Warehouse

\section{Introduction}

In the 1970s, scholars began to research DSS (Decision Support System) ${ }^{[1]}$. More and more importance is attached to decision support system in various fields, including management science, economics, applied mathematics, engineering technology, information science and so on. Researchers have put forward various decision analysis methods and solved a series of representative decision support problems through integrating several subjects and combining artificial intelligence, network technology, communication technology and information processing technology.

IDSS (Intelligent Decision Support System) is generated from the combination of DSS and AI. It is one of the hotspots of research on DSS. IDSS is an intelligent decision support system based on knowledge. Its core thought is to combine DSS and AI (Artificial Intelligence), apply expert system technology and help to solve complex decision problems through sufficiently applying the knowledge of human experts and logical reasoning. IDSS reflects the characteristic of expert system solving qualitative problems in the form knowledge reasoning, sufficiently utilizes the characteristic of decision support system solving quantitative problems with the core model calculation, combines qualitative analysis and quantitative analysis and further enhances the ability of solving problems.

\section{The classification of IDSS}

Since the 1990s, the development of Internet technology has brought challenging problems to $\mathrm{DSS}^{[2]}$. With the rapid development of distributed computing and network computing, IDSS begins to evolve and produce a serious of new concepts, ideas and structures from its centralization. According to intelligent decision method, IDSS can be divided into 3 types ${ }^{[3-4]}$ : the IDSS based on AI, the IDSS based on data warehouse and the IDSS based on case reasoning. 


\section{The IDSS based on AI}

The IDSS based on AI includes the following types:

(1) The IDSS based on ES. ES (Expert System) is a field of IDSS maturely applied at present. It is comprised of knowledge base, inference engine and database. It uses non-quantitative logical statements to express knowledge and uses automatic reasoning to solve questions. IDSS mainly uses quantification method to model problems and offers decision support utilizing the calculation result of value model.

(2) The IDSS based on machine learning. Machine learning is to get the knowledge of solving human's problems through computer simulating human's learning. Machine learning can automatically acquire knowledge. Therefore, the bottleneck of acquiring knowledge in expert system can be solved in certain degree.

(3) The IDSS based on Agent. At present, Agent is a research hotspot in AI field, including the research on intelligent Agent, the research on Mufti-Agent system and the research on Agent-oriented program design.

\section{The IDSS based on data warehouse}

Through summarizing, gathering and integrating data source information, data warehouse builds integrated, changeable and durable data set facing theme and offers useable information for making decisions. OLAP (On-line Analytical Processing) simultaneously develops with data warehouse. Through the real-time, multidimensional and complex inquiry and synthetic analysis on data warehouse, the overall characteristics and development tendency concealed in data can be known. Multidimensional data analysis of OLAP can be in the forms of stripping and slicing, rotation and drilling. IDDS architecture based on data mining is shown in Fig.1.

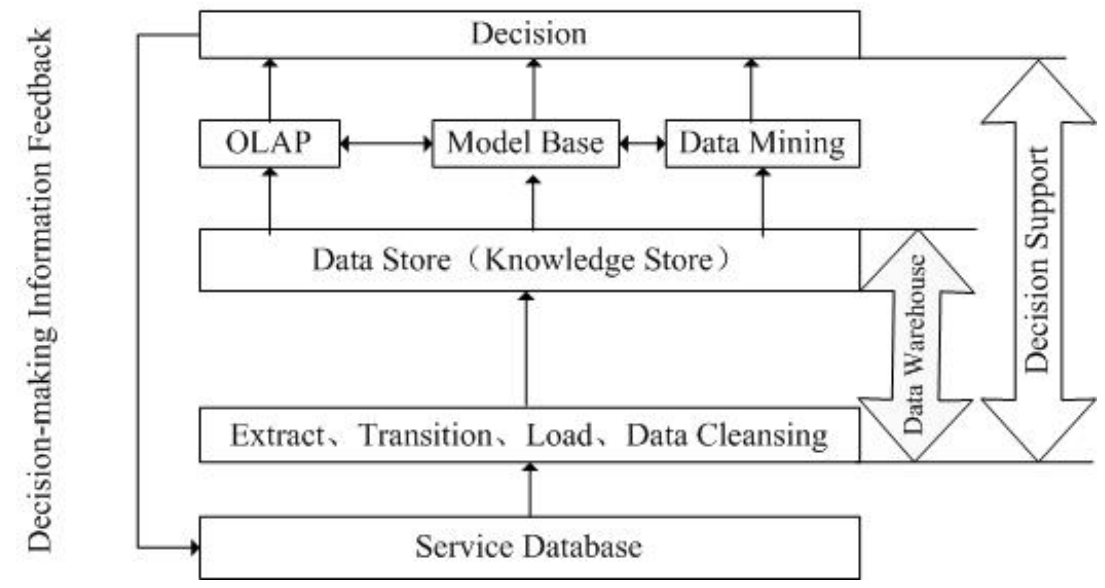

Fig.1 IDDS Architecture Based on Data Mining

\section{The IDSS based on case reasoning}

The reasoning based on case is to find the method of solving the current problems from the previous experience. The set of previous incidents can form a case base, namely the model of problems processing. The current problems are target cases; the problems or circumstances in memory are source cases. While CBR treats problems, search source cases which have the same attributes with the target cases from case base; then adjust through matching the cases. The reasoning based on case simplifies the process of acquiring knowledge, reuses previous solving process and plays good functions to the problems which can be solved through calculation. 


\section{The characteristics of IDSS}

IDSS has the following characteristics:

(1) Certain self-directed learning ability. Decision makers are permitted to modify and broaden the knowledge in knowledge base. Therefore, the ability of solving problems can be further enhanced.

(2) IDSS has reasoning mechanism. It simulates the thinking process of decision makers and applies relevant knowledge to guide decision makers to choose proper decision models through man-machine interview according to the demand of decision makers.

(3) IDSS has intelligent model management function. It manages model as a kind of knowledge structure and simplifies the interface between different subsystems.

(4) IDSS builds universal structure of decision support system to expand the service field of the system and make the system better adapt to the change of environment and the change of the form of decisions.

\section{The architecture of IDSS}

IDSS usually uses model and builds it through interaction and recursion process. It can be used by simplex user and also can be used by many users in different places based on web. Generally speaking, on the basis of the DSS of traditional model base, database and method base, knowledge base and inference engine are added to typical IDSS architecture. In addition, natural language processing system is added to man-machine conversation subsystem. Problem processing system is added between natural language processing system and the four bases of model base, database, method base and knowledge base. Just as is shown in Fig. 2.

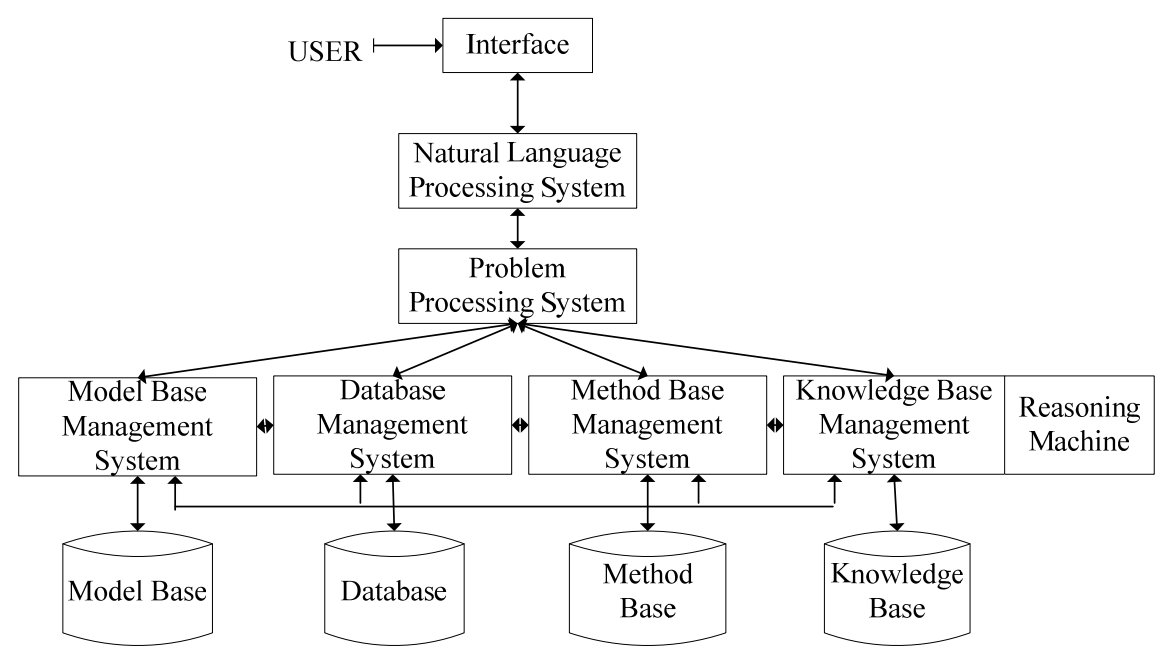

Fig.2 IDDS Architecture

\section{User interface subsystem}

The intelligent man-machine interface of the four-base system accepts the decision problems and decision goals expressed by natural language or the language which is close to natural language. This changes the performance of man-machine interface in large degree. Through user interface subsystem, users can communicate with IDSS and get the instructions of IDSS. 


\section{Problem processing system}

Problem processing system is in the central position of IDSS. It is the bridge between human and machine \& the solution resources stored in it. It is comprised of problem analyzer and problem solver.

(1) Natural language processing system: As for the problems from transformation, problem analyzer judges the structured degree of them, selects or builds model for the structured problems and uses traditional model to calculate and solve them. As for the semi-structured or non-structured problems, they can be solved by rules model and reasoning mechanism.

(2) Problem processing system: the most active part of IDSS. It can recognize and analyze problems, design solutions and invoke the data, models, methods and knowledge in the four bases for solving the problems. As for semi-structured or non-structured problems, inference engine shall be triggered for reasoning or getting new knowledge.

\section{Knowledge base subsystem and inference engine}

Knowledge management subsystem can work as an independent unit or support other subsystem to work. It offers intelligence to decision makers to enhance the ability of decision makers. This system can interconnect with knowledge base of organization which is comprised of three parts: knowledge base management system, knowledge base and inference engine.

(1) Knowledge base management system. This system meets the demand of knowledge maintenance (such as increasing, deleting and modifying the knowledge in knowledge base) and meets the demand of the knowledge needed for problem analysis and judgment in the process of making decisions.

(2) Knowledge base: the core of knowledge base subsystem. Knowledge base is used to store the expert knowledge and experience which cannot be expressed by data or models, namely the decision-making knowledge and experience of decision-making experts. The special knowledge in some specific problem fields are also included. The knowledge in the knowledge base is a group of convention for describing the world. It is the signifying process of knowledge.

(3) Inference engine. Inference engine is to elicit new facts (conclusions) from existing facts. It is a group of program and processes knowledge base (rules and facts) aiming at users' problems.

\section{Data management subsystem}

Data management subsystem is a database containing relevant data. It is managed by database management system. Data management subsystem can interconnect with data warehouse, analyze and process the knowledge in database and data warehouse through data mining and analyze multidimensional data utilizing OLAP.

\section{Model management subsystem}

Model management subsystem offers analysis ability and corresponding software management to system, including the language for building user models. Model management subsystem is a software package which includes finance, statistics, management and other quantitative models. Model management subsystem can connect commonly-used or external model storage. 


\section{Development tendency of IDSS}

IDSS develops in the directions of integration and comprehensiveness. With the development of modern science and technology, it is an important issue of IDSS to comprehensively use the advantages of key technologies (such as data warehouse, on-line analysis, data mining, model base, database, ES, object-oriented method, Agent and machine learning), form integrated decision support system and develop practical and effective IDSS. Multi-Agent system with distributed structure is an important research field of distributed intelligent decision support system (IDSS). Utilizing the characters of Multi-Agent technology, the decision problems of complex system can be solved. It offers new approaches for IDSS. As for IDSS, to get breakthrough, future IDSS design and achievement will be based on Multi-Agent for certain. Therefore, it is very significant to research the mode of developing multi-agent decision support system in new environment.

\section{Conclusions}

Human never stops exploring the nature. Decision theory has achieved prominent progress in the new era. Since the 1990s, the development of artificial intelligence, database technology and computer network technology has offered powerful technology support to the development of IDSS. With the development of science, specialties are subdivided; more and more large systems are subdivided to be complex small systems. It is a significant problem for people to use reasonable method and limited manpower and material resources to manage and guide a large and complex system. On this basis, it is firmly believed that the application prospect of IDSS will be wider.

\section{References}

[1] Gorry G A, Scott Mort on MS . A Framework for Management Information System [ J ]. Sloan Management Review , 1971, 13(1) : 55-70.

[2] Zheng Yinghua, Wu Genyou. The Model Base and Model Base Management System of Intelligent Decision Support Systems[J]. Science Technology and Engineering, 2006 (9):1312-1315.

[3] Ren Minglun, Yang Shanlin, Zhu Weidong. Intelligent Decision Support System State of Art and Challenges. Journal of System Engineering, 2002, 17(5):430 -439.

[4] Huang Ming, Tang Huanwen. A new decision support system [ J] .IEEE Intelligent Processing Systems, representation scheme for International Conference models, 1997, 2 (10):1556-1558.

[5] Cai Zixing, Wang Yong. Intelligent Systems Principles, Algorithms and Applications[M]. China Machine Press, 2014.

[6] Li Hongliang. Development of Intelligent Decision Support System and Its Application Prospect[J]. Journal of Chongqing Institute of Technology(Natural Science), 2009(10):140-144.

[7] Zhang Wei. The research review of Intelligent decision support systems[J]. Modern Business Trade Industry, 2009(14):252-253. 
[8] Liang Luoxi, Wu Jiang. Review and Prospect on Development of Decision Support System[J]. Computer Science,2016(10):27-32.

[9] Wriggers P, Kultsova M, Kapysh A, et al. Intelligent Decision Support System for River Floodplain Management[J]. Communications in Computer \& Information Science,2014,466:195-213.

[10] Dong C S J, Srinivasan A. Agent-enabled Service-oriented Decision Support Systems[J]. Decision Support Systems, 2013,55(1):364-373. 